\title{
Enhanced recovery after cystectomy in patients with preoperative narcotic use
}

Saum Ghodoussipour, MD; Alireza Ghoreifi, MD; Behdod Katebian, MD; Brian H. Cameron, MD; Anirban P. Mitra, MD, PhD; Jie Cai, MS; Gus Miranda, BS; Anne K. Schuckman, MD; Siamak Daneshmand, MD; Hooman Djaladat, MD, MS Institute of Urology, Norris Comprehensive Cancer Center, University of Southern California, Los Angeles, CA, United States

Cite as: Ghodoussipour A, Ghoreifi A, Katebian B, et al. Enhanced recovery after cystectomy in patients with preoperative narcotic use. Can Urol Assoc J 2021 May 11; Epub ahead of print. http://dx.doi.org/10.5489/cuaj.7007

Published online May 11, 2021

Correspondence: Dr. Hooman Djaladat, Institute of Urology, USC/Norris Comprehensive Cancer Center, Los Angeles, CA, United States; djaladat@med.usc.edu

$* * *$

\section{Abstract}

Introduction: The aim of this study was to evaluate the outcomes of radical cystectomy with an enhanced recovery after surgery (ERAS) protocol in patients with a history of chronic preoperative narcotic use compared to narcotic-naive patients.

Methods: We identified 553 patients who underwent open radical cystectomy with ERAS. Preoperative narcotic use was identified in 34 patients who were then matched to 68 narcoticnaive patients. Postoperative outcomes, opioid use, and visual analog scale (VAS) pain scores were analyzed and compared. All routes of opioid use were recorded and converted to a morphine equivalent dose (MED).

Results: Patients with preoperative narcotic use reported higher median VAS pain scores per day (POD1: 5.2 vs. 3.9, $\mathrm{p}=0.003$; POD2: 5.1 vs. 3.6, $\mathrm{p}<0.001$; POD3: 4.6 vs. 3.8, $\mathrm{p}=0.004$ ) and used significantly more opioids (median MED) per day (POD1: 13.2 vs. 10.0, $\mathrm{p}=0.02$; POD2: 11.3 vs. $6.4, p=0.003$; POD3: 10.2 vs. $5.0, p=0.005$ ) following surgery. Preoperative narcotic users were noted to have a significantly higher incidence of 90 -day readmissions $(41.2 \% \mathrm{vs} .20 .6 \%$, $\mathrm{p}=0.03$ ). There was no difference in median hospital stay ( 4 vs. 4 days, $\mathrm{p}=0.6$ ), 30- or 90 -day complications $(64.7 \%$ vs. $60.3 \%, p=0.8$ and $82.4 \%$ vs. $75.0 \%, p=0.4$, respectively) or gastrointestinal complications $(29.4 \%$ vs. $26.5 \%, \mathrm{p}=0.8)$, including postoperative ileus $(11.8 \%$ vs. $20.6 \%, p=0.2$ ). 
Conclusions: Patients with preoperative narcotic exposure report higher pain scores and require more opioid use following radical cystectomy with ERAS and are more likely to be readmitted within 90 days. However, there was no observed difference in hospital stay or complications.

\section{Introduction}

With an estimated 81,400 new cases diagnosed in 2020, bladder cancer is one of the most common malignancies in the United States (U.S.). Moreover, 17,980 patients are estimated to die from bladder cancer in 2020, making it one of the most lethal urologic diseases. ${ }^{1}$ Radical cystectomy (RC) with urinary diversion is the gold standard management for patients with muscle invasive or high-risk non-muscle invasive disease. $\mathrm{RC}$ is a morbid procedure with historical early complication rates of up to $67 \%$ and length of stay of up to 9-11 days., ${ }^{2,3}$ The perioperative management of patients undergoing $\mathrm{RC}$ is complex and has seen major improvement in recent years owed to the increased acceptance of enhanced recovery after surgery (ERAS) protocols. ${ }^{4}$ We have previously shown the benefit of an ERAS protocol in reducing length of stay without an increase in complications or readmission rates. ${ }^{5}$ ERAS protocols are multimodal pathways that streamline all elements of perioperative care, including pre, intra, and post-operative interventions. A major focus is on the minimization of narcotic pain management and the use of $\mu$ opioid antagonists to accelerate gastrointestinal recovery. ${ }^{6}$ As we strive to improve ERAS protocols and subsequent outcomes, we must target all modifiable factors and better understand non-modifiable ones. In 2017, the U.S. Department of Health and Human Services declared an opioid epidemic. ${ }^{7}$ Over 2 million patients abuse opioids yearly, with an annual incidence of more than 200,000 new users and 16,000 deaths from opioid overdose. ${ }^{8}$ The crisis is widespread and affects many patients undergoing urologic procedures including RC. The efficacy of modern treatment practices on patients with a history of preoperative narcotic use needs to be better understood. In this study, we investigate the effect of preoperative narcotic use on outcomes in patients undergoing RC with an enhanced recovery protocol.

\section{Methods}

\section{Study population}

Using our prospectively maintained, institutional review board-approved bladder cancer database, we identified all patients with preoperative narcotic use who underwent open RC, pelvic lymph node dissection and urinary diversion for urothelial carcinoma with intent to cure from June 2012 to June 2017, the initial 5 years after implementation of our ERAS protocol. Preoperative narcotic users were defined as those having an active prescription for narcotics for $\geq 30$ days prior to surgery. Narcotic users were identified through preoperative history and physical documentation and meticulous chart review was then performed. Inactive prescriptions 
and those without verified indications were excluded. Preoperative narcotic users were then matched 1:2 to narcotic naïve patients after controlling for age, gender, pathologic tumor stage and urinary diversion (orthotopic vs heterotopic).

\section{Pain management protocol}

Our ERAS protocol has been described previously. ${ }^{5}$ The pain management component of the protocol at the time of the study included intraoperative use of intravenous (IV) acetaminophen and ketorolac, postoperative around-the-clock oral acetaminophen, IV ketorolac, and infusion of ropivacaine via para-incisional subfascial catheters. Oral and IV narcotics were reserved for breakthrough pain only. Oral pain medications were initiated on postoperative day (POD) 0 and patients were converted to oral-only analgesics by POD 3.

\section{Data collection}

A dedicated database manager records patient and disease characteristics, complications and details of readmissions in our prospectively maintained database. Complications were classified using the Clavien-Dindo system; grade 1 and 2 complications were grouped as "minor," and grades 3- 5 were grouped as "major" complications. ${ }^{3}$ Complications were also categorized by organ system. Gastrointestinal (GI) complications included diarrhea, bowel obstruction, postoperative ileus (POI), constipation and GI bleeding. POI was defined as nausea or vomiting with abdominal distension that required cessation of oral intake, nasogastric tube placement, or intravenous fluid therapy. ${ }^{6}$ Infectious complications included urinary tract infection, sepsis, pneumonia, and clostridium difficile. Renal/electrolyte complications included dehydration, electrolyte abnormalities, acute kidney injury, and hydronephrosis.

In-hospital opioid use and visual analogue scale (VAS) pain scores were analyzed retrospectively. Pain scores were averaged from each hour during inpatient hospital stay to calculate a daily average, which was then averaged across the entire hospital stay to achieve a mean VAS score for that hospital admission. Opioid administration by all routes was obtained through meticulous chart review and converted to a morphine equivalent dose (MED). These ratios were obtained through previous studies. ${ }^{9-11}$ To avoid overestimation, the lowest epiduralto-intravenous ratios were used.

\section{Data analysis}

SAS Version 9.4 (SAS Institute Inc., Cary, NC, USA) was used for all data analyses. Pearson's chi-square or Fisher's exact tests were used to examine the association between categorical demographic, clinical and pathologic variables. Kruskal-Wallis test was used to assess differences between continuous variables that were not normally distributed. All $p$-values are 2 -sided and $\mathrm{p}<0.05$ was considered statistically significant. 


\section{Results}

A total of 722 open radical cystectomies were performed during the study period. Of these, 553 were for curative intent and enrolled in the ERAS protocol. Preoperative narcotic use was identified in 34 (6.09\%) patients. Indications for preoperative narcotic use included chronic arthritic conditions in $16(47.1 \%)$, neurologic disease in $3(8.8 \%)$, urologic related pain in 9 $(26.5 \%)$ and other causes in $6(17.6 \%)$. Narcotic users were matched to 68 patients with no reported history of narcotic use. Demographic characteristics of the matched cohorts are summarized in Table 1.

\section{Pain scores and in-hospital narcotic use}

Preoperative narcotic users reported a higher median VAS pain score than narcotic naïve patients on each postoperative day (POD1; 5.2 vs 3.9, $\mathrm{p}=0.003$, POD2; 5.1 vs 3.6, $\mathrm{p}<0.001$, POD3; 4.6 vs $3.8, \mathrm{p}=0.004)$. VAS pain scores tended to decrease as patients approached discharge from the hospital (Figure 1). In addition, preoperative narcotic users received significantly more opioids (median MEDs) per postoperative day (POD1; 13.2 vs 10.0, $\mathrm{p}=0.020$, POD2; 11.3 vs 6.4, $\mathrm{p}=0.003$, POD3; 10.2 vs $5.0, \mathrm{p}=0.005$ ). Morphine equivalent dosing also tended to decrease as patients approached discharge from the hospital (Figure 2).

\section{Outcomes}

Preoperative narcotic users were noted to have a lower estimated blood loss during their operations (mean $462.3 \pm 350.0 \mathrm{ml}$ vs $523.8 \pm 318.4 \mathrm{ml}, \mathrm{p}=0.04$ ). There was no significant difference in operative time (mean $5.5 \pm 1.5$ hours vs $5.6 \pm 1.2$ hours, $\mathrm{p}=0.170$ ) or median length of stay ( 4 days vs 4 days, $p=0.6$ ) between the two groups.

On univariate analysis, we did not find a significant difference in rate of complications between preoperative narcotic users and narcotic naïve patients at 30 days $(64.7 \% \mathrm{vs} 60.3$, $\mathrm{p}=0.8$ ) or 90 -days $(82 \%$ vs $75 \%, \mathrm{p}=0.4)$. There was similarly no significant difference in GI complications $(29.4 \%$ vs $26.5 \%, p=0.8)$ or POI $(11.8 \%$ vs $20.6 \%, p=0.2)$. Cardiac, pulmonary, and infectious complications were similar between the groups (Table 2), but preoperative narcotic users did have a higher rate of renal/electrolyte-related $(41.2 \%$ vs $20.6 \%, p=0.03)$ and bleeding-related complications $(26.5 \%$ vs $7.4 \%, \mathrm{p}=0.01)$.

Readmissions were similar at 30 days $(20.6 \%$ vs $13.2 \%, \mathrm{p}=0.3)$, but preoperative narcotic users did have a higher rate of 90-day readmissions when compared to narcotic naïve patients (41.2\% vs 20.6\%, $\mathrm{p}=0.03$ ). Complications leading to 90 -day readmissions were stratified based on etiology and are summarized in Table 3. Narcotic users were noted to have a higher proportion of major complications requiring 90-day readmission compared to narcotic naïve patients $(50.0 \%$ vs $28.6 \%$, respectively, $\mathrm{p}=0.26)$, though not significantly so. The most common complications resulting in readmission amongst both groups were renal/electrolyte causes (dehydration), followed by infectious and then GI-related causes. 


\section{Discussion}

The opioid epidemic exposes urologists to more and more patients with a history of narcotic use before surgery. Chronic preoperative narcotic use has been shown to be associated with higher complications and worse functional outcomes following non-urological surgeries, ${ }^{12-14}$ though the data is sparse in urology literature including radical cystectomy. Given the complexity and associated morbidity with radical cystectomy and urinary diversion, more information in this unique patient subset is needed. Previous studies have shown that ERAS protocols not only decrease hospital stay, but also decrease in-hospital pain, narcotic use and GI complications when compared to traditional recovery protocols. ${ }^{5,9,15}$ In a prior report on pain management following RC at our institution, we found that ERAS patients used significantly less opioids (4.9 vs 20.7 MEDs, $\mathrm{p}<0.001$ ) and had a lower rate of POI when compared to a pre-ERAS cohort ( $7.3 \%$ vs $22 \%, p=0.03) .{ }^{9}$ However, we excluded narcotic users in that study. In the current study, our primary goal was to explore the effects of an ERAS protocol in patients with a preoperative history of narcotic use, specifically to determine if they experience the same benefits as those patients without a history of recent narcotic use.

To compare in-hospital narcotic requirements, a MED was used. ${ }^{11}$ We found that patients with preoperative narcotic use had significantly more postoperative pain than narcotic naïve patients and required significantly more opioids following surgery. These results are in line with prior studies that suggest opioids may induce hyperalgesia $(\mathrm{OIH}) \cdot{ }^{16-18} \mathrm{Chu}$ et al. provided evidence for the development of OIH when they tested 6 patients after one month of oral morphine therapy for lower back pain and found significant hyperalgesia to experimental cold pain. ${ }^{16}$ While the methodology of studies testing OIH vary, the mechanism is thought to be due to neuroplastic changes in the peripheral and central nervous systems that lead to sensitization of pronociceptive pathways. ${ }^{17}$ Ren et al. performed a study in 54 opiate addicts and 46 healthy controls and noted hyperalgesia in the addicts. They also found that pain distress was positively correlated with opiate craving. ${ }^{18}$ The association between exaggerated pain responses and drug craving may explain the findings in our study.

Despite the higher pain scores and greater in-hospital opioid use in preoperative narcotic users, there was no significant difference in clinical outcomes aside from renal/electrolyte-related complications and readmissions within 90 days. There were more high-grade complications leading to readmission in the preoperative narcotic users compared to narcotic naïve patients, but we were unable to identify a significant difference probably due to the limited sample size of readmitted patients. The majority of readmissions in preoperative narcotic users were due to the high-grade renal/electrolyte complications (35.7\% compared to $21.4 \%$ in the narcotic naïve group). This could be related to the effect of narcotics on the small and large bowel transit that will be resulted in the increase of fluid absorption, third spacing, and fluid/electrolyte imbalance. A recent report showed 142,557 emergency department visits for opioid overdose in the U.S. in 2017 alone, ${ }^{19}$ but we were unable to attribute any of our readmissions to opioid overdose. 
Interestingly, we did not find significant differences in GI complications amongst our two groups. A focus on GI-related complications is particularly important as the link between narcotic use and bowel function is clear. Previous studies have shown that an increased daily dose of narcotics and increasing days on narcotics both predict POI and length of stay. ${ }^{10,20,21}$ The overall GI complication rate in our study was $29.4 \%$ in preoperative narcotic users and $26.5 \%$ in narcotic naïve patients $(\mathrm{p}=0.82)$, while rate of POI was $11.8 \%$ and $20.6 \%(\mathrm{p}=0.26)$ in the respective groups.

Another reason to emphasize GI complications is that prior studies suggest that these represent the most common reason for prolonged length of stay after radical cystectomy. ${ }^{22}$ The median length of stay in both groups was 4 days and not different from prior reports from our institutional ERAS protocol. ${ }^{5}$ While the previously referenced studies found increased POI and length of stay in narcotic users or those who received greater narcotics in hospital, our patient cohort is unique as all patients were treated within an ERAS protocol. There are various measures in our protocol that aim to mitigate postoperative stressors, specifically GI-related ones. There is evidence to support many of these measures including lack of bowel preparation, no nasogastric tube, and early feeding. ${ }^{23-25}$ However, the strongest evidence-based intervention in our protocol is the use of pre- and peri-operative alvimopan, a $\mu$ opioid receptor antagonist that has been shown to decrease rates of POI, length of stay, and cost after radical cystectomy. ${ }^{26,27}$ Alvimopan was approved by the U.S. Food and Drug Administration (FDA) in 2008 to accelerate GI recovery following partial large or small-bowel resection with primary anastomosis. ${ }^{28}$ However, this approval came with a risk evaluation and mitigation strategy (REMS) label warning against its use in chronic narcotic users, defined as those who have been receiving opioids for $>7$ consecutive days. Thus, the only randomized controlled trial investigating alvimopan after RC excluded patients who had used narcotics preoperatively. ${ }^{24}$ This REMS label came from preliminary results of a phase 3, double blind, placebo-controlled study that aimed to evaluate the long-term safety of alvimopan in patients with non-cancer related opioid induced bowel dysfunction (OBD). This study enrolled 805 patients with OBD and randomized them $2: 1$ to 538 receiving alvimopan $0.5 \mathrm{mg}$ twice daily vs 267 who received placebo. The most common adverse events in the study were GI related, including abdominal pain and diarrhea ( $40 \%$ for alvimopan and $35 \%$ for placebo). Serious adverse events occurred in $13 \%$ of patients treated with alvimopan and in $11 \%$ with placebo, but 7 patients $(1.3 \%)$ in the alvimopan arm suffered from myocardial infarction compared to 0 with placebo. The overall rate of serious cardiovascular (CV) events was $2.6 \%$ with alvimopan and $1.12 \%$ with placebo. All $\mathrm{CV}$ events occurred in patients with established or at high risk for CV disease. Still, these findings led to a hold on the drug by the FDA, which was temporary as other studies investigating the safety of alvimopan in OBD showed a myocardial infarction rate of $0.08 \%$ with alvimopan compared to $0.38 \%$ with placebo and all serious CV events $1.18 \%$ with alvimopan vs $0.96 \%$ with placebo. ${ }^{29-31}$ The first of these three studies examined 168 patients with OBD 
(minimum one month of opioid therapy) and found that alvimopan increased the proportion of patients having a bowel movement (BM) within 8 hours of starting the drug $(54 \%, 43 \%$, and $29 \%$ for alvimopan $1 \mathrm{mg}, 0.5 \mathrm{mg}$ and placebo, respectively, $\mathrm{p}<0.001) .{ }^{29}$ The latter two studies randomized 1040 patients with OBD to alvimopan or placebo and found a significant increase in the rate of weekly BMs ( $<<0.001$ for both studies). ${ }^{30,31}$ An FDA panel later declared that the issue of CV safety was not class specific for $\mu$ opioid antagonists ${ }^{32}$ and we have safely administered alvimopan to all patients enrolled in our ERAS protocol, irrespective of prior narcotics use. There was no difference in the cardiac complication rate in this study at $7.4 \%$ for narcotic naïve and $11.8 \%$ for preoperative narcotic exposed patients $(p=0.48)$.

While the cause of increased 90-day readmissions cannot be explained by the results of our study, we do feel our results show that the cumulative effects of the measures included in our ERAS protocol level the playing field for patients with preoperative narcotic use such that they experience similar lengths of stay and complications, importantly GI-related ones, compared to narcotic naïve patients. The greater pain experienced by patients with preoperative narcotic use is an area for improvement and one that we have targeted with multidisciplinary management with our anesthesia colleagues. A recent change to our ERAS protocol since the time of this study has been multimodal prophylactic pain management with oral acetaminophen, celecoxib and gabapentin for 3 days prior to surgery. We are also trying to further improve outcomes through preoperative education sessions and potentially more effective therapeutics.

Our study is limited by the retrospective nature of preoperative narcotic use determination. We defined narcotic use before surgery as having an active prescription for $\geq 30$ days, but we do not know how consistently patients were taking narcotics. Similarly, many patients who abuse narcotics may do so without a prescription. ${ }^{33}$ A prospective identification of preoperative narcotic users could allow for quantities of preoperative narcotics to be recorded. Still our results provide a useful reference for surgeons who treat patients with the common scenario of a known but un-quantified history of narcotic use prior to surgery. Another limitation is that VAS scores are currently recorded at inconsistent time intervals and there is no way to collect objective pain information. However, this issue is not limited to our study and must be considered in any study assessing pain management. The strength of this study is to present a group of patients who underwent homogenous cystectomy and received similar perioperative care with as high as $88 \%$ compliance rate to our ERAS protocol. ${ }^{34}$ Nevertheless, this sample is not representative of the complete ERAS cohort at our institution and includes only those with preoperative narcotics use and their matched cohort. Moreover, we did not evaluate differences in opioid consumption after discharge from the hospital. Such information will be useful to urologists as we combat the opioid epidemic.

\section{Conclusions}

We found that patients with a history of preoperative narcotic use experience more pain and need higher doses of narcotics following radical cystectomy. Preoperative narcotic use may increase 
the risk of 90-day readmissions but does not affect the rate of GI complications or hospital stay following radical cystectomy. 


\section{References}

1. Siegel RL, Miller KD, Jemal A. Cancer statistics, 2020. CA Cancer J Clin 2020;70:7-30. doi: 10.3322/caac.21590.

2. Kim SP, Shah ND, Karnes RJ, et al. The implications of hospital acquired adverse events on mortality, length of stay and costs for patients undergoing radical cystectomy for bladder cancer. J Urol 2012;187:2011-7. doi: 10.1016/j.juro.2012.01.077.

3. Shabsigh A, Korets R, Vora KC, et al. Defining early morbidity of radical cystectomy for patients with bladder cancer using a standardized reporting methodology. Eur Urol 2009;55:164-74. doi: 10.1016/j.eururo.2008.07.031.

4. Tyson MD, Chang SS. Enhanced Recovery Pathways Versus Standard Care After Cystectomy: A Meta-analysis of the Effect on Perioperative Outcomes. Eur Urol 2016;70:995-1003. doi: 10.1016/j.eururo.2016.05.031.

5. Daneshmand S, Ahmadi H, Schuckman AK, et al. Enhanced recovery protocol after radical cystectomy for bladder cancer. J Urol 2014;192:50-5. doi: 10.1016/j.juro.2014.01.097.

6. Bazargani ST, Djaladat H, Ahmadi H, et al. Gastrointestinal Complications Following Radical Cystectomy Using Enhanced Recovery Protocol. Eur Urol Focus 2018;4:889-94. doi: 10.1016/j.euf.2017.04.003.

7. HHS.gov. HHS acting secretary declares public health emergency to address national opioid crisis. [released 2017 Oct 26]. https://www.hhs.gov/.

8. Florence CS, Zhou C, Luo F, et al. The Economic Burden of Prescription Opioid Overdose, Abuse, and Dependence in the United States, 2013. Med Care 2016;54:901-6. doi: 10.1097/MLR.0000000000000625.

9. Xu W, Daneshmand S, Bazargani ST, Cai J, et al. Postoperative Pain Management after Radical Cystectomy: Comparing Traditional versus Enhanced Recovery Protocol Pathway. J Urol 2015;194:1209-13. doi: 10.1016/j.juro.2015.05.083.

10. Cali RL, Meade PG, Swanson MS, Freeman C. Effect of Morphine and incision length on bowel function after colectomy. Dis Colon Rectum 2000;43:163-8. doi: 10.1007/BF02236975.

11. Nielsen S, Degenhardt L, Hoban B, et al. A synthesis of oral morphine equivalents (OME) for opioid utilisation studies. Pharmacoepidemiol Drug Saf 2016;25:733-7. doi: 10.1002/pds.3945.

12. Yerneni K, Nichols N, Abecassis ZA, et al. Preoperative Opioid Use and Clinical Outcomes in Spine Surgery: A Systematic Review. Neurosurgery 2020;86:E490-E507. doi: 10.1093/neuros/nyaa050.

13. Gan T, Jackson NA, Castle JT, et al. A Retrospective Review: Patient-Reported Preoperative Prescription Opioid, Sedative, or Antidepressant Use Is Associated with Worse Outcomes in Colorectal Surgery. Dis Colon Rectum 2020;63:965-73. doi: 10.1097/DCR.0000000000001655.

14. Hilliard PE, Waljee J, Moser S, et al. Prevalence of Preoperative Opioid Use and Characteristics Associated With Opioid Use Among Patients Presenting for Surgery. JAMA Surg 2018;153:929-37. doi: 10.1001/jamasurg.2018.2102. 
15. Frees SK, Aning J, Black P, et al. A prospective randomized pilot study evaluating an ERAS protocol versus a standard protocol for patients treated with radical cystectomy and urinary diversion for bladder cancer. World J Urol 2018;36:215-20. doi: 10.1007/s00345-017-2109-2.

16. Chu LF, Clark DJ, Angst MS. Opioid tolerance and hyperalgesia in chronic pain patients after one month of oral morphine therapy: a preliminary prospective study. J Pain 2006;7:43-8. doi: 10.1016/j.jpain.2005.08.001.

17. Lee M, Silverman SM, Hansen H, et al. A comprehensive review of opioid-induced hyperalgesia. Pain Physician 2011;14:145-61.

18. Ren ZY, Shi J, Epstein DH, et al. Abnormal pain response in pain-sensitive opiate addicts after prolonged abstinence predicts increased drug craving. Psychopharmacology 2009;204:423-9. doi: 10.1007/s00213-009-1472-0.

19. Vivolo-Kantor AM, Seth P, Gladden RM, et al. Vital Signs: Trends in Emergency Department Visits for Suspected Opioid Overdoses - United States, July 2016-September 2017. MMWR Morb Mortal Wkly Rep 2018;67:279-85. doi: 10.15585/mmwr.mm6709e1.

20. Barletta JF, Asgeirsson T, Senagore AJ. Influence of intravenous opioid dose on postoperative ileus. Ann Pharmacother 2011;45:916-23. doi: 10.1345/aph.1Q041.

21. Goettsch WG, Sukel MP, van der Peet DL, et al. In-hospital use of opioids increases rate of coded postoperative paralytic ileus. Pharmacoepidemiol Drug Saf 2007;16:668-74. doi: $10.1002 /$ pds. 1338 .

22. Chang SS, Baumgartner RG, Wells N, et al. Causes of increased hospital stay after radical cystectomy in a clinical pathway setting. J Urol 2002;167:208-11.

23. Tabibi A, Simforoosh N, Basiri A, et al. Bowel preparation versus no preparation before ileal urinary diversion. Urology 2007;70:654-8. doi: 10.1016/j.urology.2007.06.1107.

24. Packiam VT, Agrawal VA, Pariser JJ, et al. Redefining the implications of nasogastric tube placement following radical cystectomy in the alvimopan era. World J Urol 2017;35:625-31. doi: 10.1007/s00345-016-1910-7.

25. Voskuilen CS, van de Putte EEF, der Hulst JB, et al. Short-term outcome after cystectomy: comparison of early oral feeding in an enhanced recovery protocol and feeding using Bengmark nasojejunal tube. World J Urol 2018;36:221-29. doi: 10.1007/s00345-017-2133-2.

26. Kauf TL, Svatek RS, Amiel G, et al. Alvimopan, a peripherally acting $\mu$-opioid receptor antagonist, is associated with reduced costs after radical cystectomy: economic analysis of a phase 4 randomized, controlled trial. J Urol 2014;191:1721-7. doi: 10.1016/j.juro.2013.12.015.

27. Lee CT, Chang SS, Kamat AM, et al. Alvimopan accelerates gastrointestinal recovery after radical cystectomy: a multicenter randomized placebo-controlled trial. Eur Urol 2014;66:265-72. doi: 10.1016/j.eururo.2014.02.036.

28. Hughes B. 2008 FDA drug approvals. Nat Rev Drug Discov 2009;8:93-6. doi: $10.1038 / \mathrm{nrd} 2813$.

29. Paulson DM, Kennedy DT, Donovick RA, et al. Alvimopan: an oral, peripherally acting, mu-opioid receptor antagonist for the treatment of opioid-induced bowel dysfunction--a 21-day treatment-randomized clinical trial. J Pain 2005;6:184-92. doi:

10.1016/j.jpain.2004.12.001. 
30. Jansen JP, Lorch D, Langan J, et al. A randomized, placebo-controlled phase 3 trial (Study SB-767905/012) of alvimopan for opioid-induced bowel dysfunction in patients with non-cancer pain. J Pain 2011;12:185-93. doi: 10.1016/j.jpain.2010.06.012.

31. Webster L, Jansen JP, Peppin J, et al. Alvimopan, a peripherally acting mu-opioid receptor (PAM-OR) antagonist for the treatment of opioid-induced bowel dysfunction: results from a randomized, double-blind, placebo-controlled, dose-finding study in subjects taking opioids for chronic non-cancer pain. Pain 2008;137:428-40. doi: 10.1016/j.pain.2007.11.008.

32. Anderson P. FDA Panel Debates CV Safety of Opioid Constipation Drugs. Medscape2014. [released 2013 June 13]. https://www.medscape.com/.

33. Voon P, Karamouzian M, Kerr T. Chronic pain and opioid misuse: a review of reviews. Subst Abuse Treat Prev Policy 2017;12:36. doi: 10.1186/s13011-017-0120-7.

34. Ghodoussipour S, Naser-Tavakolian A, Cameron B, et al. Internal audit of an enhanced recovery after surgery protocol for radical cystectomy. World J Urol 2020;38:3131-37. doi: $10.1007 / \mathrm{s} 00345-020-03135-\mathrm{w}$. 


\section{Figures and Tables}

Fig 1. Postoperative visual analog scale (VAS) pain scores in the two groups. POD: postoperative day.

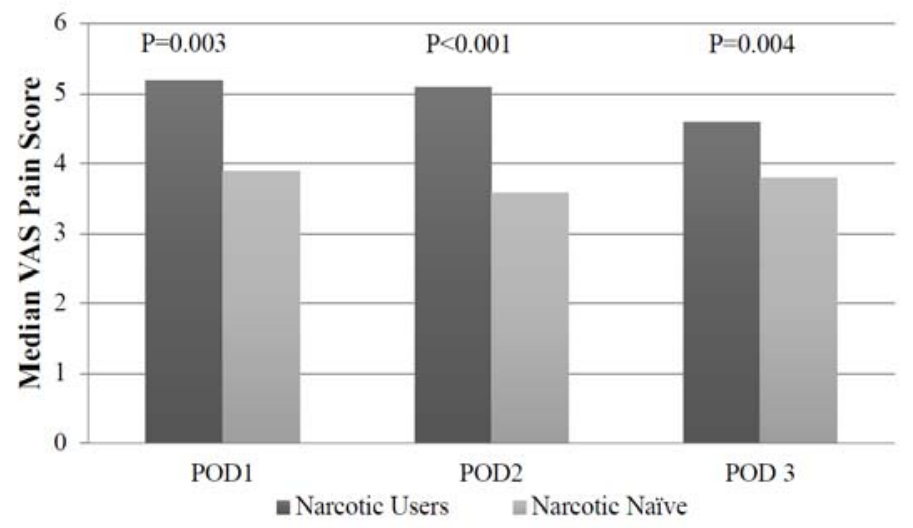

Fig 2. Postoperative narcotic use in the two group. POD: postoperative day.

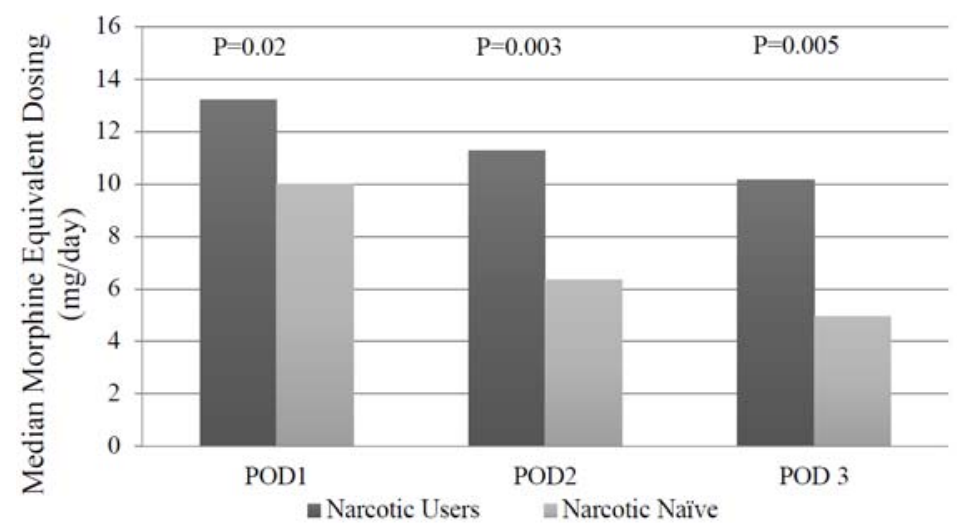




\begin{tabular}{|c|c|c|c|}
\hline Variable & Narcotic-naive & Narcotic user & \\
\hline & $n=68$ & $\mathrm{n}=34$ & $\mathbf{p}$ \\
\hline \multicolumn{3}{|l|}{ Gender, n (\%) } & 1.00 \\
\hline Male & $52(76.5 \%)$ & $26(76.5 \%)$ & \\
\hline Median age (IQR) & $70.5(65-79)$ & $71(64-76)$ & 0.81 \\
\hline Median BMI (IQR) & $27.2(24.9-31)$ & $25.2(21.5-28.6)$ & 0.02 \\
\hline \multicolumn{3}{|l|}{ CCI, n (\%) } & 0.36 \\
\hline \multirow{3}{*}{$\begin{array}{l}0 \\
1 \\
\geq 2\end{array}$} & $30(44.1 \%)$ & $10(29.4 \%)$ & \\
\hline & $13(19.1 \%)$ & $8(23.5 \%)$ & \\
\hline & $25(36.8 \%)$ & $16(47.1 \%)$ & \\
\hline ASA class, $n(\%)$ & 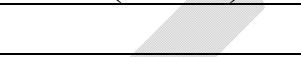 & & 0.15 \\
\hline $1-2$ & $7(10.3 \%)$ & $7(20.6 \%)$ & \\
\hline $3-4$ & $61(89.7 \%)$ & $27(79.4 \%)$ & \\
\hline Neoadjuvant chemotherapy, n (\%) & $26(38.2 \%)$ & $11(32.4 \%)$ & 0.56 \\
\hline Prior abdominal surgery, $\mathrm{n}(\%)$ & $8(11.9 \%)$ & $4(11.8 \%)$ & 0.37 \\
\hline Prior pelvic radiation, $\mathrm{n}(\%)$ & $1(1.5 \%)$ & $2(5.9 \%)$ & 0.21 \\
\hline Tumor stage, n (\%) & & & 0.97 \\
\hline \multirow{3}{*}{$\begin{array}{l}<\mathrm{pT} 3 \mathrm{N0} \\
\geq \mathrm{pT} 3 \mathrm{~N} 0 \\
\mathrm{~N}+\end{array}$} & $48(70.6 \%)$ & $24(70.6 \%)$ & \\
\hline & $11(16.2 \%)$ & $5(14.7 \%)$ & \\
\hline & $9(13.2 \%)$ & $5(14.7 \%)$ & \\
\hline \multicolumn{3}{|l|}{ Diversion type, n (\%) } & 0.88 \\
\hline Orthotopic & $43(63.2 \%)$ & $22(64.7 \%)$ & \\
\hline Heterotopic & $25(36.8 \%)$ & $12(35.3 \%)$ & \\
\hline
\end{tabular}

ASA: American Society of Anesthesiologists; CCI: Charlson comorbidity index; IQR: interquartile range. 


\begin{tabular}{|c|c|c|c|}
\hline Outcomes, n (\%) & Narcotic-naive & Narcotic user & \\
\hline & $n=68$ & $n=34$ & $\mathbf{p}$ \\
\hline 30-day complications (overall) & $41(60.3)$ & $22(64.7)$ & 0.8 \\
\hline Low-grade & $36(52.9)$ & $18(52.9)$ & 0.7 \\
\hline High-grade & & $5(7.4)$ & $4(11.8)$ \\
\hline 90-day complications (overall) & $51(75.0)$ & $28(82.4)$ & 0.4 \\
\hline Low-grade & $41(60.3)$ & $18(52.9)$ & \multirow[t]{2}{*}{0.2} \\
\hline High-grade & $10(14.7)$ & $10(29.4)$ & \\
\hline 30-day readmissions & $9(13.2)$ & $7(20.6)$ & 0.3 \\
\hline 90-day readmissions & $14(20.6)$ & $14(41.2)$ & 0.03 \\
\hline $\begin{array}{l}\text { Gastrointestinal } \\
\text { complications }\end{array}$ & $18(26.5)$ & $10(29.4)$ & 0.8 \\
\hline Postoperative ileus & $14(20.6)$ & $4(11.8)$ & 0.2 \\
\hline $\begin{array}{l}\text { Renal/electrolyte } \\
\text { complications }\end{array}$ & $14(20.6)$ & $14(41.2)$ & $\mathbf{0 . 0 3}$ \\
\hline Infectious complications & $21(30.9)$ & $11(32.4)$ & 1.00 \\
\hline $\begin{array}{l}\text { Thromboembolic } \\
\text { complications }\end{array}$ & $6(8.8)$ & $3(8.8)$ & 1.00 \\
\hline Cardiac complications & $5(7.4)$ & $4(11.8)$ & 0.4 \\
\hline Pulmonary complications & $1(1.5)$ & $3(8.8)$ & 0.1 \\
\hline Neurological complications & $3(4.4)$ & $3(8.8)$ & 0.4 \\
\hline
\end{tabular}

*All complication subtypes are recorded within 90 days following surgery 


\begin{tabular}{|l|c|c|c|c|c|c|}
\hline \multicolumn{3}{|l}{$\begin{array}{l}\text { Table 3. Minor and major complications requiring 90-day readmissions by category in 14 } \\
\text { narcotic-naive and 14 preoperative narcotic users undergoing radical cystectomy with ERAS }\end{array}$} \\
\hline $\begin{array}{l}\text { Complication requiring } \\
\text { readmission at 90-days, } \\
\mathbf{n}(\%)\end{array}$ & $\begin{array}{c}\text { Minor } \\
\text { Narcotic- } \\
\text { naive }\end{array}$ & $\begin{array}{c}\text { Narcotic } \\
\text { users }\end{array}$ & $\mathbf{p}$ & $\begin{array}{c}\text { Major } \\
\text { users }\end{array}$ & $\begin{array}{c}\text { Narcotic- } \\
\text { naive }\end{array}$ & p \\
\hline Total & $10(71.4)$ & $7(50.0)$ & 0.26 & $7(50.0)$ & $4(28.6)$ & 0.26 \\
\hline Gastrointestinal & $3(21.4)$ & $1(7.1)$ & 0.30 & $0(0)$ & $0(0)$ & - \\
\hline Renal/electrolyte & $3(21.4)$ & $3(21.4)$ & 1.00 & $5(35.7)$ & $3(21.4)$ & 0.42 \\
\hline Infectious & $3(21.4)$ & $2(14.3)$ & 0.64 & $1(7.1)$ & $0(0.0)$ & 0.33 \\
\hline Thromboembolic & $0(0)$ & $0(0)$ & - & $1(7.1)$ & $1(7.1)$ & 1.00 \\
\hline Cardiac & $1(7.1)$ & $0(0)$ & 0.33 & $0(0)$ & $0(0)$ & - \\
\hline
\end{tabular}

